\title{
Société Canadienne d'Études de la Renaissance / Canadian Society for Renaissance Studies
}

Congrès annuel de la SCER/CSRS.

Le IVe congrès de la S.C.E.R. eut lieu du 23 au 25 mai 1979 à l'Université de Saskatchewan à Saskatoon. Les sessions académiques ont porté sur l'histoire, la littérature et la Réforme. La journée du 23 mai fut consacrée entièrement aux communications de caractère historique. En accordant à l'histoire une place importante dans le congrès de Saskatoon, on a voulu mettre en relief la contribution importante du département d'histoire de l'Université de Saskatchewan à l'avancement des études européennes en général et aux travaux sur la Renaissance en particulier par la publication de la revue Canadian Journal of History/Annales Canadiennes d'Histoire. L'organisateur Claude Sutto de l'Université de Montréal a réussi à nous présenter des contributions qui étaient en même temps variées et complémentaires. Sous la présidence de Peter Bietenholz de l'Université de Saskatchewan nous avons entendu les communications suivantes:

Christopher Stocker (Univ. of British Columbia), "Political Strùctures and Political Space in Sixteenth Century France: Town, Crown and Religious "Parties" in the 1560's." En examinant en détail le cas de la ville d'Orléans dans ses rapports avec le parti de Condé et avec la monarchie, le professeur Stocker a démontré que les structures existantes et le sens de loyauté ont joué un rôle déterminant dans l'évolution politique de la France pendant les premières guerres de religion.

Egmont Lee (Un. of Calgary), "The political society of Città di Castello: a computer study." Le professeur Lee nous a offert un excellent exemple de l'utilisation de l'ordinateur dans le domaine de l'histoire en nous présentant une étude de la petite ville italienne Città di Castello pendant la période qui précède et suit la chute de Nicolo Vitelli (1467-1482). Au delà des analyses quantitative et statistique, l'utilisation de l'ordinateur lui a permis de retrouver la complexité de l'administration citadine et une connaissance approfondie de la société. E. Lee insiste égallement sur la complémentarité des études historiques fondées sur des sources narratives traditionnelles et des données obtenues par l'utilisation de l'ordinateur.

André Vermeirre (Montréal), "Pertinence d'une étude historique globale de l'espace 
mer du Nord-mer Baltique." En prenant ses distances vis-d-vis de l'histoire nationale, André Vermeirre a fait ressortir l'intérêt pour une approche nouvelle et globale sur l'histoire des "peuples du Nord." Pour l'espace Nord-mer Baltique, il n'existe rien de comparable aux travaux de Fernand Braudel sur la Méditerranée. André Vermeirre nous propose une étude extrêmement saisissante pour la période de la fin du Moyen-Age et de la Renaissance et Réforme.

Claire Dolan (Univ. Laval), “Attitudes collectives et représentations des notables au XVIe siècle: la notion d'étranger à Aix-en-Provence vue à travers le phénomène protestant." Par une lecture sérielle des documents du Conseil municipal d'Aixen-Provence, le professeur Dolan nous a offert un exemple concret d'histoire des mentalités en faisant ressortir les attitudes collectives à l'égard de l'étranger et du protestant.

Daniel Hickey (Univ. de Moncton), "The crisis of the nobility revisited." Ses réexamens de la crise de la noblesse française à partir du cas du dauphinais font ressortir la non-existence d'une véritable crise de la vieille noblesse; d'autre part les nouveaux nobles ne sont pas tellement les riches bourgeois et les détenteurs des offices publics mais les militaires qui ont combattu pendant les guerres de religion.

Au cours de la session du 24 au matin consacrée à la Littérature (in the Context of Ideas and the other Arts), organisée et présidée par David Hoeniger (Victoria College, Un. of Toronto), nous avons écouté quatre conférences extrêmement suggestives:

Patricia Vicari (Un. of Toronto), "Orpheus and Orphism in the Renaissance."

Alice Rathé (Un. of Toronto), "Le Cappiccio dans les écrits de Vasari."

Martin Bergbusch (Regina, Sask.), "Sovereignty in Sidney's New Arcadia."

Vernon McCarthy (Regina, Sask.), "Renaissance Lyric: 'The World needs such music' (Herrick and Lawes)."

La session du 25 au matin, organisée et présidée par Gerald Hobbs (Vancouver School of Theology), portait sur la Réforme. Les trois conférences ont donné lieu à de nombreux échanges.

Les rapports entre la peinture et la Réforme ont été exposés par Rosemarie Bergmann (McGill University), “A 'trostlich pictura': Luther's Attitude in the Question of Images."

Jane Abray (Un. of Western Ontario), a présenté: "The fate of the French Protestant community in Strasbourg during the religious wars."

La conférence de Bernard Roussel (Faculté de Théologie protestante, Strasbourg, France), "Probleme biblique et réveil religieux en France entre 1525 et 1545," a soulevé de nombreuses hypothèses de recherches pour l'étude de la diffusion de 


\section{4 / Renaissance and Reformation}

la Bible par les Réformés français. Nous devons signaler que la présence du professeur Roussel aux différentes activités de notre congrès s'est révélée extrêmement précieuse.

Le banquet et la rencontre sociale, devenus de tradition dans nos congrès, furent une grande réussite grâce à la prévoyance et à l'hospitalité du professeur Peter Bietenholz et de son épouse Doris. Après un repas fraternel à l'Université, la famille Bietenholz nous a acceuilli dans un cadre enchanteur et une ambiance de la Renaissance dans laquelle nous avons eu la satisfaction d'écouter un excellent concert de musique de la Renaissance par un ensemble d'étudiants et de professeurs. Si notre congrès de Saskatoon fut un succès c'est en grande partie grâce à la contribution de Peter et Doris Bietenholz.

J.M. DE BUJANDA, Université de Sherbrooke 